\title{
Amino Acid Uptakes in Normal and Small-for-Date Placentas, with Special Reference to Activity of Transport ATPase in Placental Slices and Isolated Trophoblastic Membrane
}

\author{
Ryuji Yamaguchi, Tetsunori Matsuda and Tomoko \\ AIZAWA \\ Department of Obstetrics and Gynecology, Nara Medical \\ University, Kashihara, Nara 634
}

Yamaguchi, R., Matsuda, T. and Aizawa, T. Amino Acid Uptakes in Normal and Small-for-Date Placentas, with Special Reference to Activity of Transport ATPase in Placental Slices and Isolated Trophoblastic Membrane. Tohoku J. exp. Med., 1978, 125 (4), 367-376-In our previous report, we presumed that there is a depressed transport of amino acids in the small-for-date (SFD) placenta. To substantiate this presumption, we conducted in vitro experiments with normal and SFD placental slices and compared their uptakes of amino acids, serine and lysine. Activity of $\mathrm{Na}-\mathrm{K}$ ATPase in the trophoblastic membrane was also studied. (1) In both normal and SFD placentas, uptakes of amino acids were about two to three times as great for neutral amino acid serine as for basic amino acid lysine. (2) In the SFD placenta, amino acid uptake became significantly slower than in the normal after $30 \mathrm{~min}$ of incubation. After $90 \mathrm{~min}$ the uptake was lower than the normal by some $32 \%$ for serine and $42 \%$ for lysine. (3) Serine uptake began to be interrupted by the addition of $10^{-3} \mathrm{M}$ ouabain at $30 \mathrm{~min}$, and showed about $31 \%$ decrease at $90 \mathrm{~min}$, revealing an amino acid uptake pattern similar to that of the SFD placenta. But lysine uptake was not noticeably affected by ouabain. (4) As for the release of intracellular amino acids into the incubation medium, no differences could be observed either between serine and lysine or between the normal and the SFD placenta. (5) Activity of transport ATPase of the isolated trophoblastic membrane was lower in the SFD than in the normal placenta. The findings tend to corroborate the suggestion that the low $\mathrm{f} / \mathrm{m}$ ratio of amino acids in SFD pregnancy accounts for a decreased placental transport of amino acids corresponding with depression in the activity of its major promotor - membrane-bound transport ATPase. —_ placenta; amino acid transport; ATPase

In our previous clinical study of the placental transport of amino acids in search of the cause of small-for-date (SFD) pregnancy, we found that the concentration of amino acids was lower in the umbilical vein blood than in the

Received for publication, January 5, 1978.

Chief: Prof. Ryuji Yamaguchi

Supported by Grant from the Ministry of Health and Welfare, Japan, for Researches on Handicapped Children, 1977; and Grant No. 148267 from the Ministry of Education, Science and Culture, Japan, 1977.

Reprint request: Ryuji Yamaguchi, M.D., Department of Obstetrics and Gynecology, Nara Medical University, Kashihara, Nara 634, Japan. 
maternal vein blood in SFD pregnancy, implying a reduced ability of the SFD placenta to transport amino acids (Matsuda et al. 1977). In this report, we newly conducted a study to find a clue to understanding the SFD identity, with in vitro experiments carried out with intracellular uptakes of marker amino acids in the human placenta and on the activity of trophoblastic membrane-bound transport ATPase (Na-K ATPase), which is generally considered to concern the active transport of amino acids.

\section{Materials and Methods}

Placentas used in the experiments were from 37 cases of normal and 19 of maternaldisease-free SFD pregnancy delivered at our Obstetric Clinic.

\section{Uptake and release of amino acids}

Uptake of amino acids

Preparation of placental specimens. Immediately after delivery, the placenta was promptly taken out and washed thoroughly in physiological saline at $0^{\circ} \mathrm{C}$, and then saline was removed by filter-paper. After blood vessels were removed as far as possible, $3 \sim 4 \mathrm{~g}$ of the placental pieces were subjected to a preincubation at $37^{\circ} \mathrm{C}$ for $10 \mathrm{~min}$ in a solution of Krebs-Ringer bicarbonate $(\mathrm{pH} 7.4)$ under an aerobic condition $\left(95 \% \mathrm{O}_{2}+5 \%\right.$ $\mathrm{CO}_{2}$ ). Then water was removed by filter-paper, and placental specimen thus processed was sliced at about $100 \mathrm{mg}$ wet weight apiece.

Incubation. As marker amino acids were used serine, L- $\left[{ }^{3} \mathrm{H}(\mathrm{G})\right]$ specific activity $1 \mathrm{Ci} /$ mmole, and lysine, L- $\left[{ }^{3} \mathrm{H}(\mathrm{G})\right]$ specific activity $1 \mathrm{Ci} / \mathrm{mmole}$. Each marker amino acid was adjusted by $0.01 \mathrm{~N} \mathrm{HCl}$ to approximately $100,000 \mathrm{cpm}$ per $100 \mu \mathrm{l}$, and to this was added some $100 \mathrm{mg}$ of placental slice and $5 \mathrm{ml}$ of Krebs-Ringer solution. The mixture was incubated at $37^{\circ} \mathrm{C}$ under an aerboic condition for a scheduled time. Measurement of intracellular and extracellular spaces was made employing the inulin space method (Dancis et al. 1968). Inulin, $\left[{ }^{3} \mathrm{H}(\mathrm{G})\right]$ specific activity $500 \mathrm{mCi} / \mathrm{g}$, was adjusted to about $100,000 \mathrm{cpm}$ per $100 \mu \mathrm{l}$ and was incubated for $1 \mathrm{hr}$ under the same condition as for the marker amino acids. The calculation was made using the following formula:

$$
\text { Concentration in ICS }=\frac{\text { total } \mathrm{cpm} \text { in tissue }-\mathrm{cpm} / \mathrm{ml} \text { in medium ECS }}{\text { total tissue water-ECS }} \text {, }
$$

where ICS and ECS are intracellular and extracellular spaces. Total tissue water was obtained by subtracting the dry weight (dried at $50^{\circ} \mathrm{C}$ for $96 \mathrm{hr}$ ) from the wet weight.

Release of amino acids from the placental tissue to the incubation medium

After incubation for $60 \mathrm{~min}$ under the described condition, the placental slice was washed for a short time in a solution of $0.154 \mathrm{M} \mathrm{NaCl}$ at $0^{\circ} \mathrm{C}$. Then water was removed by filter-paper, and the placental slice was incubated in an amino acid-free Krebs-Ringer solution for 15 and $30 \mathrm{~min}$ under the aerobic condition. The proportion of release into the medium was expressed by percentage of $\mathrm{cpm}$ in the medium to the total of that in the medium and tissue.

\section{Radioactivity count}

The placental tissue was processed with a Packard tri-carb sample oxidizer, and $100 \mathrm{ml}$ of dioxane scintillator (PPO $4.5 \mathrm{~g}$, POPOP $4.5 \mathrm{mg}$ and naphthalin $75 \mathrm{~g}$ in a blend composed of $363 \mathrm{ml}$ of ethanol, $330 \mathrm{ml}$ of dioxane and $330 \mathrm{ml}$ of xylene, totaling $1,023 \mathrm{ml}$ ) were added to $0.5 \mathrm{ml}$ of the incubation medium. Radioactivity was counted by employing the Packard tri-carb-liquid scintillation spectrometer.

\section{Experiment on the trophoblastic membrane}

\section{Preparation of trophoblastic membrane specimens}

Immediately after the placenta was taken out, the umbilical arteries and vein were 
cannulated to let 100,000 units of heparin sodium solution flow through the vessels. Then some 4 liters of $0.154 \mathrm{M} \mathrm{NaCl}$ were perfused through the vessels for $30 \mathrm{~min}$ to remove blood. After that, the placenta on the maternal side was taken off and cut into small pieces by scissors in an ice bath. These placental pieces were left in place for $30 \mathrm{~min}$ in a solution of $1 \mathrm{mM}$ of $\mathrm{NaHCO}_{3}$ containing $2 \mathrm{mM}$ of $\mathrm{CaCl}_{2}$. Then the suspension was homogenized using the Dounce homogenizer (clearance $0.15 \sim 0.2 \mathrm{~mm}$ ) to obtain trophoblastic membrane specimens by employing a modification of the method of Emmelot et al. (1964) (Fig. 1).

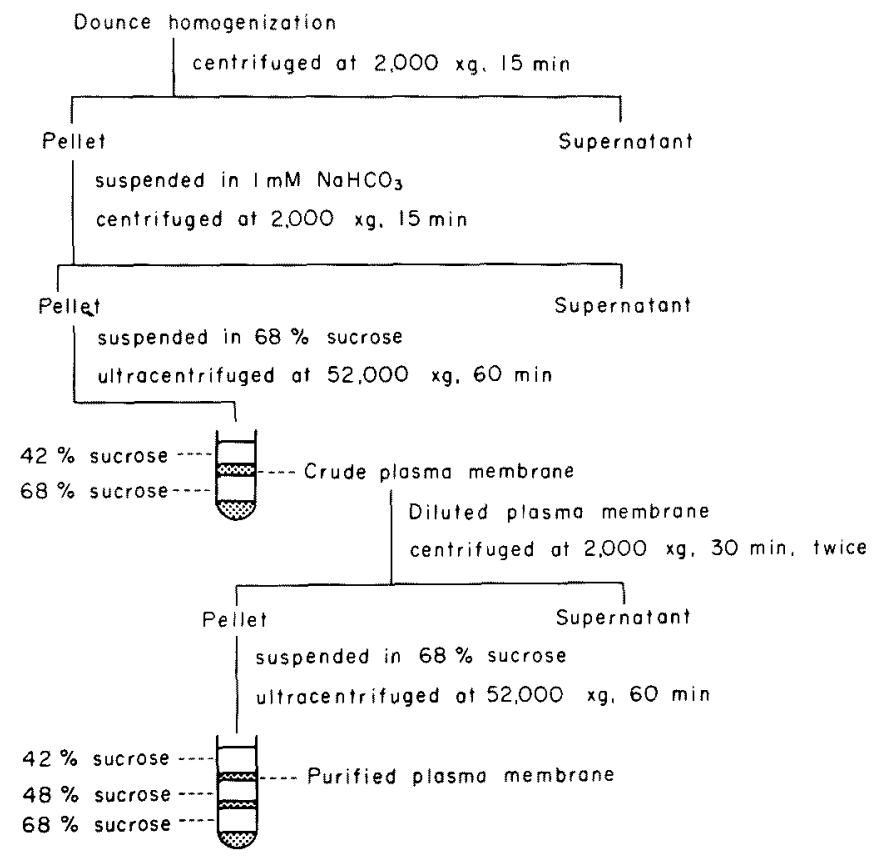

Fig. 1. Isolation scheme for the trophoblastic plasma membrane of the human term placenta (Emmelot et al. 1964).

Determination of transport ATPase (Na-K ATPase)

$100 \mu \mathrm{l}$ of the membrane preparation blend were added to $0.8 \mathrm{ml}$ of $20 \mathrm{mM}$ Tris- $\mathrm{HCl}$ (pH 7.4) containing $3 \mathrm{mM}$ Tris-ATP, $5 \mathrm{mM} \mathrm{MgCl}, 140 \mathrm{mM} \mathrm{NaCl}$ and $14 \mathrm{mM} \mathrm{KCl}$, and were incubated at $37^{\circ} \mathrm{C}$ for a scheduled time. $30 \mathrm{~min}$ after the reaction was stopped by adding $1 \mathrm{ml}$ of $10 \%$ trichloroacetic acid, the blend was filtered. To $1 \mathrm{ml}$ of the filtrate were added $0.5 \mathrm{ml}$ of $10 \mathrm{~N} \mathrm{H}_{2} \mathrm{SO}_{4}, 0.5 \mathrm{ml}$ of $2.5 \%\left(\mathrm{NH}_{4}\right)_{6} \mathrm{MoO}_{7} \mathrm{O}_{2}$ and $2 \mathrm{ml}$ of distilled water, and further $0.05 \mathrm{ml}$ of a mixture composed of $0.2 \mathrm{~g}$ of 1-amino-2-naphthol-4-sulfonic acid, $1.2 \mathrm{~g}$ of $\mathrm{NaHSO}_{3}$ and $1.2 \mathrm{~g}$ of $\mathrm{Na}_{2} \mathrm{SO}_{4}$ per $100 \mathrm{ml}$. After the mixture was let alone for 30 min, inorganic phosphorus ( $\mathrm{Pi}$ ) in it was quantitated using Fiske-Subbarow's method (Fiske and Subbarow 1925). The quantity of Pi determined in the $\mathrm{Mg}^{++}$-free incubation medium was subtracted from that in the $\mathrm{Mg}^{++-a d d e d}$ medium, and the difference was decided as Na-K ATPase. As the standard $\mathrm{Pi}, \mathrm{NaH}_{2} \mathrm{PO}_{4}$ was used.

Determination of glucose-6-phosphatase

To examine comparative purity of the isolated trophoblastic membrane specimen, glucose-6-phosphatase (G-6-Pase), which is a marker of contamination of organelle components, was determined using Nordlie's method (Nordlie and Arion 1966). The chemical reaction in the blend of trophoblastic membrane specimens was stopped by adding, 
to $100 \mu \mathrm{l}$ of the blend, $0.6 \mathrm{ml}$ of $0.1 \mathrm{M}$ cacodylate buffer $(\mathrm{pH} 6.5), 200 \mu \mathrm{l}$ of $0.15 \mathrm{M}$ sodium glucose-6-phosphate, and $0.6 \mathrm{ml}$ of distilled water. Then inorganic phosphorus (Pi) was quantitated using Fiske-Subbarow's method. Quantitation of protein was made by Lowry's method (Lowry et al. 1951).

Electron-microscopic observation

In order to assess the purity of trophoblastic membrane specimen, the membrane preparation was fixed in $2 \%$ glutaraldehyde in phosphate buffer (pH 7.4) and subsequently fixed in $1 \%$ aqueous osmium tetroxide solution. Thus processed sections were stained with uranyl acetate and lead, and observed with a Hitachi HU-11D electron microscope.

\section{Results}

Amino acid uptake in the placental slice

Fig. 2 shows the uptakes of neutral amino acid serine and basic amino acid lysine in the normal and the SFD placenta. In both placental slices, serine uptake was two to three times greater than lysine uptake at each scheduled time of measurement.

The uptakes of two marker amino acids were only slightly lower in the SFD placenta than in the normal 15 min after incubation was initiated, but at $30 \mathrm{~min}$ and later the uptakes turned significantly lower in the SFD placenta $(p<0.01)$, showing a decrease rate of $32 \%$ for serine and $42 \%$ for lysine at $90 \mathrm{~min}$.

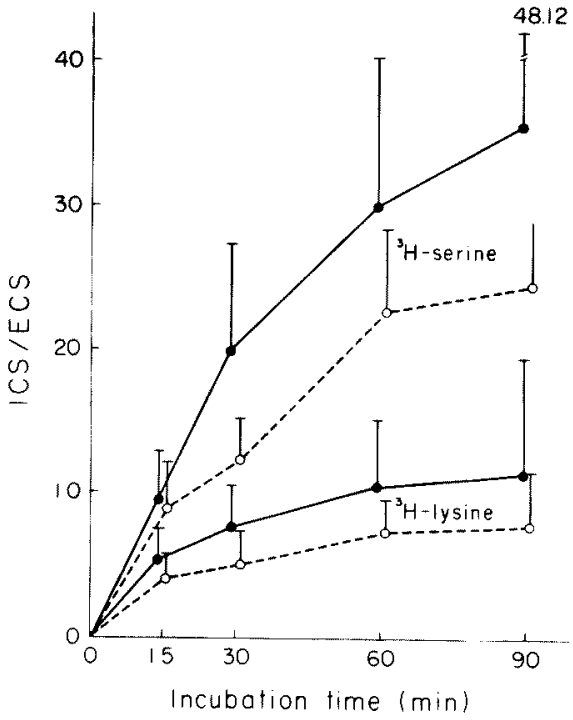

Fig. 2

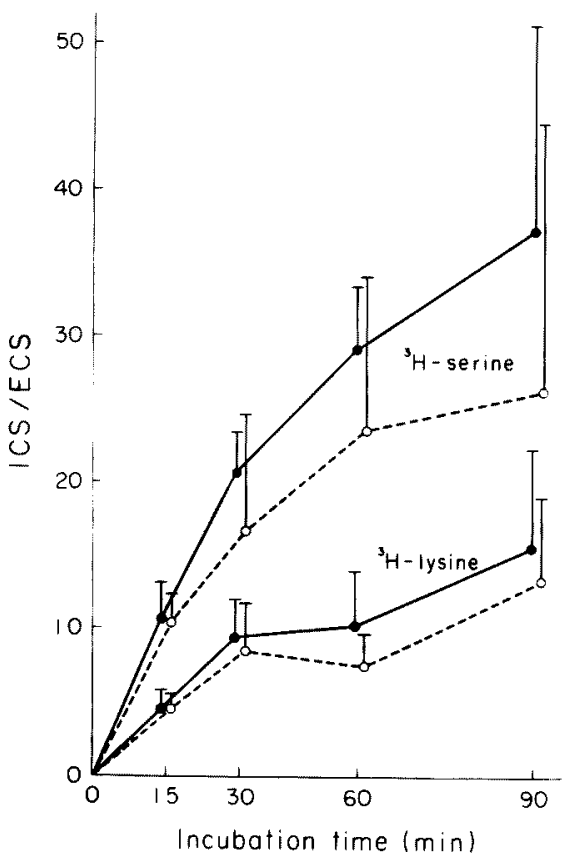

Fig. 3

Fig. 2. Difference of amino acid uptake between the normal and SFD placentas in vitro. $\bullet-\bullet$, normal $(n=26)$; o-, SFD $(n=9)$.

Fig. 3. Effects of ouabain blocking on intracellular uptakes of serine and lysine. normal $(n=7)$; $0-0$, ouabain $(n=7)$. 
Changes in amino acid uptake of the normal placenta with and without addition of $10^{-3} \mathrm{M}$ ouabain are shown in Fig. 3. No noticeable differences were brought about by the addition of ouabain at $15 \mathrm{~min}$ of incubation. But later, serine in the ouabain-added group showed a distinctly lower rate of uptake than in the control, indicating a significant difference $(p<0.025)$ at $60 \mathrm{~min}$ and as much as some $31 \%$ decrease at $90 \mathrm{~min}$. In contrast to serine, the uptake of lysine was not affected significantly by ouabain.

Amino acid release from the placental slice to the medium

Table 1 shows distinctive features of amino acid release from the placental slice into the incubation medium. The release of serine increased with time, but

TABLE 1. Release rate of amino acids from placental slices into medium

\begin{tabular}{|c|c|c|c|c|}
\hline & \multicolumn{2}{|c|}{${ }^{3} \mathrm{H}$-serine } & \multicolumn{2}{|c|}{${ }^{3} \mathrm{H}$-lysine } \\
\hline & $15 \mathrm{~min}$ & $30 \mathrm{~min}$ & $15 \mathrm{~min}$ & $30 \mathrm{~min}$ \\
\hline $\begin{array}{l}\text { Normal } \\
\text { placenta } \\
(n=10)\end{array}$ & $33.7 \pm 5.3$ & $45.7 \pm 7.2$ & $42.2 \pm 9.8$ & 41. $2 \pm 8.4$ \\
\hline $\begin{array}{l}\text { SFD } \\
\text { placenta } \\
(n=5)\end{array}$ & $37.2 \pm 6.6$ & $46.6 \pm 5.2$ & $46.0 \pm 10.0$ & $46.7 \pm 14.1$ \\
\hline
\end{tabular}

Release rate $(\%)=\frac{\mathrm{cpm} \text { in medium }}{\mathrm{cpm} \text { in medium and tissue }}$

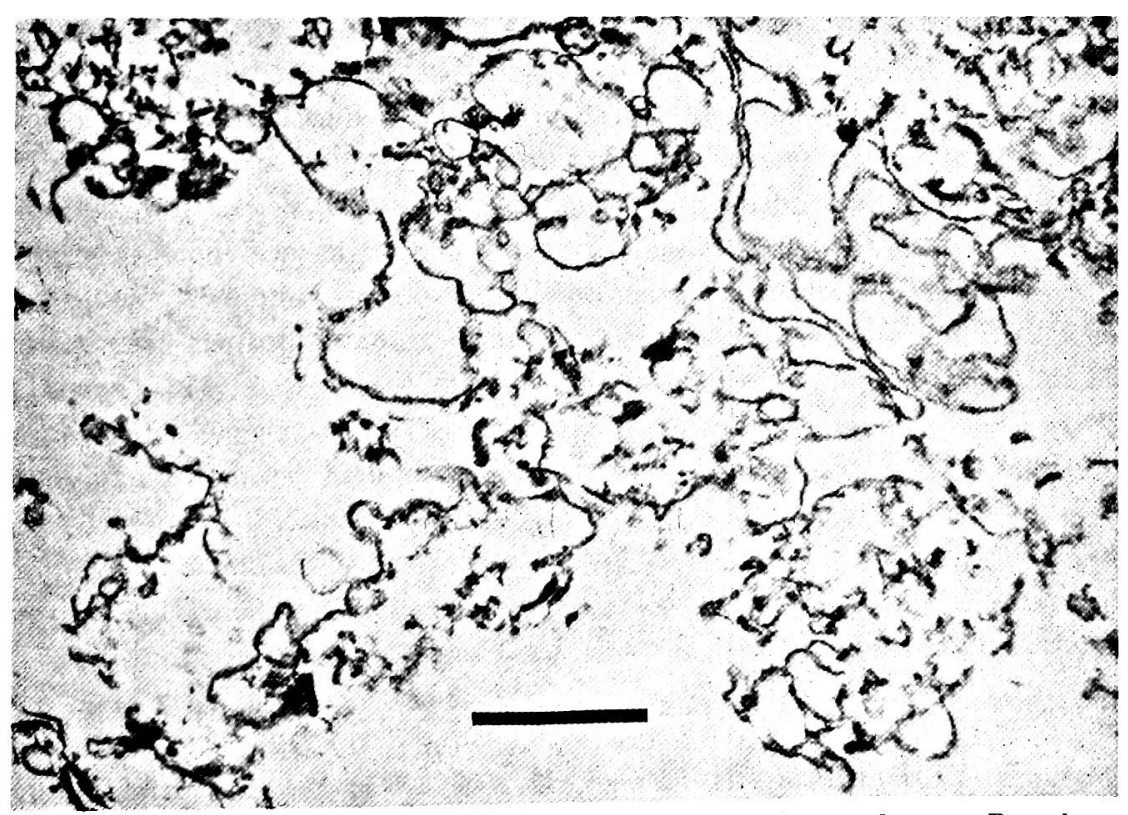

Fig. 4. Electron microscopic finding of isolated trophoblastic membrane. Bar shows 1 $\mu \mathrm{m}$. 
that of lysine attained a plateau $15 \mathrm{~min}$ after the initiation of incubation. The release rate was slightly higher for lysine than for serine at $15 \mathrm{~min}$, though the difference was not significant; at $30 \mathrm{~min}$ release rates of two amino acids were almost equivalent. Compared with the normal placenta, the SFD placenta showed slightly higher release rates of both serine and lysine at 15 and $30 \mathrm{~min}$, though either difference was not significant.

Transport ATPase activity of the isolated trophoblastic membrane specimen

The comparative purity of the trophoblastic membrane material was examined electron-microscopically (Fig. 4) and biochemically (Table 2). The ratio in ATPase activity (Pi released per minute calculated from $10 \mathrm{~min}$ incubation) of the membrane to the whole tissue was 3.2 in the normal placenta and 2.7 in the

TABLE 2. Enzyme activities of isolated membrane specimen

\begin{tabular}{|c|c|c|c|c|}
\hline & & Na-K ATPase & $\mathrm{G} \cdot 6 \cdot \mathrm{Pas}$ & \\
\hline Normal placenta & $\begin{array}{l}\mathrm{M} \\
\mathrm{W} \\
\mathrm{M} / \mathrm{W}\end{array}$ & $\left.\begin{array}{r}10.6 \pm 2.5 \\
3.7 \pm 1.4 \\
3.2 \pm 1.1^{*}\end{array}\right\}(n=7)$ & $\left.\begin{array}{r}10.2 \pm 7.0 \\
6.8 \pm 3.7 \\
1.6 \pm 0.8^{*}\end{array}\right\}$ & $(n=8)$ \\
\hline SFD placenta & $\begin{array}{l}\mathrm{M} \\
\mathbf{W} \\
\mathbf{M} / \mathrm{W}\end{array}$ & $\left.\begin{array}{l}5.5 \pm 2.3 \\
2.7 \pm 1.5 \\
2.7 \pm 2.0^{*}\end{array}\right\}(n=8)$ & $\left.\begin{array}{l}7.4 \pm 4.5 \\
6.6 \pm 3.4 \\
1.2 \pm 0.5^{*}\end{array}\right\}$ & $(n=6)$ \\
\hline
\end{tabular}

Figures indicate the rate of $\mathrm{Pi}$ liberation in $\mu \mathrm{g} / \mathrm{mg}$ protein $/ \mathrm{min}$ determined in 10 minutes' incubation.

* Mean \pm s.D. was calculated from each $M / W$ value.

M, membrane; W, whole tissue.

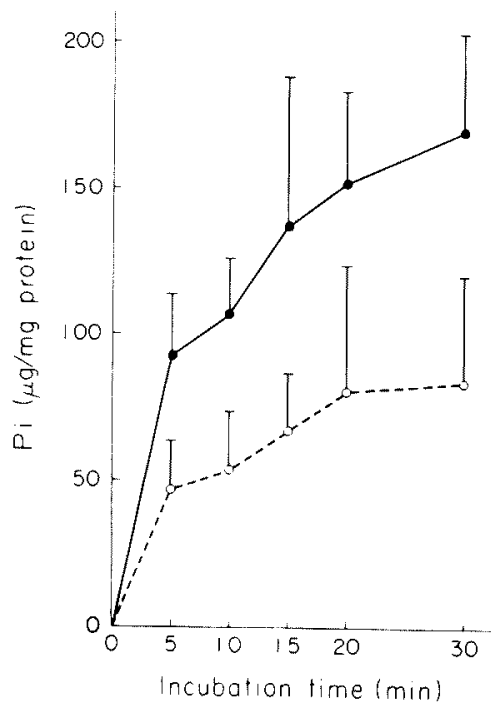

Fig. 5. $\mathrm{Na}^{+}-\mathrm{K}^{+}$ATPase activity of isolated trophoblastic membranes from normal and SFD placentas. $\bullet-$, normal $(n=11)$; $0-0$, SFD $(n=10)$. 
SFD placenta. The corresponding ratio in G-6-Pase was 1.6 and 1.2, respectively. These values were not compatible with those reported in other organs $(10 \sim 15$ in ATPase and 0.5 1 in G-6-Pase). These discrepancies may suggest that our membrane specimens were not completely pure but rather crude containing some organelle components. And also, our results may be attributable to the characteristic structure of the placenta that the epithelium is mainly composed of inseparable syncytiotrophoblasts.

The differences between the normal and the SFD placenta in the activity feature of Na-K ATPase in the trophoblastic membrane are shown in Fig. 5. The activity increased gradually with time in like manner in both placentas. But the level of activity in SFD was only half as high as that in the normal placenta at each scheduled time of measurement, with a difference invariably significant $(p<0.05)$.

\section{Discussion}

In our previous study, we found a lower $\mathrm{f} / \mathrm{m}$ ratio of blood amino acids in SFD pregnancy with no maternal diseases, and suggested a probability that the active transport of amino acids is depressed in the SFD placenta (Matsuda et al. 1977). In an attempt to substantiate this probability, we conducted experiments of tissue slice incubation. Although this method has a difficulty in indicating the direction of transport of substances, we adopted it in this study according to the opinion of Miller and Berndt (1977) that the amino acid uptake in the placental slice is somehow indicative of the transport more inclining from mother to fetus.

The features of placental uptakes of serine and lysine were different. Christensen et al. (1973), experimenting with Ehrlich ascites tumor cells, stated that neutral amino acid serine is transported by the $\mathrm{Na}^{+}$-dependent system, while diamino acid lysine is transported by an unknown system, probably with the aid of neutral amino acid. This may suggest that the transport processes of serine and lysine are different also in the placenta. Enders et al. (1976) showed no difference in the uptake of amino acids between the placenta and other organs.

Regarding the total amount of amino acids absorbed in the placenta, Schneider and Dancis (1974), experimenting with placental slices, observed that after $90 \mathrm{~min}$ of incubation, the intracellular (ICS) concentrations of serine and lysine are about 20 times and 2 times as high as the corresponding values of the extracellular spaces (ECS). Higher distinctions between ICS and ECS were found in our data, i.e. 35 times for serine and 13 times for lysine. The differences between the results of Schneider and Dancis and the present study are perhaps due to different experimental methods. Smith et al. (1973) and Gusseck et al. (1975) reported that the longer the pre-incubation period of the placental slice, the intracellular uptakes of amino acids are the greater.

Whether do our data indicate an example of active transport of amino acids in the placenta or not? Miller and Berndt (1974), using $\alpha$-amino-isobutyric acid (AIB), carried out experiments under varying conditions of $\mathrm{pH}$, temperature, 
aerobic atomosphere, and with specimen groups added ouabain or 2,4-dinitrophenol (DNP). They showed that amino acid uptake in the placenta represents active transport depending on $\mathrm{Mg}^{++}$ion and $\mathrm{Na}-\mathrm{K}$ ATPase. Similar results were shown by other authors; Litonjua et al. (1967) in their experiments under aerobic and anaerobic atomosphere, Dancis et al. (1968) through experiments with metabolic inhibitors such as DNP and cyanide with the use of AIB, and Gusseck et al. (1975) with protein synthesis inhibitors. Our experiment with ouabain seems to present an instance of active transport of serine, showing a $31 \%$ decrease at 90 min. A greater decrease of $58 \%$ in the corresponding figure was shown by Sybulski and Tremblay (1967) in their experiment with glycine. The difference may be due to the dissimilarity in the concentration of ouabain used (theirs $0.07 \mathrm{mM}$ ) as well as in the marker amino acid (glycine against serine). On the other hand, ouabain addition less affected lysine uptake, showing only an insignificant decrease. This may account for the difference in transport mode as mentioned above.

It is very suggstive that in the SFD placenta, the uptake of serine and lysine became significantly reduced at $30 \mathrm{~min}$ and later. It is notable that the figures are close to those observed in the $10^{-3} \mathrm{M}$ ouabain-added normal placenta. This tends to strengthen our previous presumption that the low $\mathrm{f} / \mathrm{m}$ ratio of blood amino acids in SFD pregnancy would account for a depressed active transport of amino acids in the SFD placenta, and emphasizes the possibility that such depression is attributable to the depressed activity of membrane-bound Na-K ATPase.

It is important to discuss in what manner amino acids once taken up in the placental tissues are released into the medium. The presumption prior to the experiment was that: (1) the release of amino acids into the medium would be greater from the normal placenta than from the SFD placenta, and (2) the amount of release would be greater for lysine than for serine inasmuch as the previous clinical data showed the $\mathrm{f} / \mathrm{m}$ ratio higher for lysine than for serine, and the present data indicated intracellular uptakes two- to three-fold greater for serine than for lysine. Contrary to the presumption, the actual results disclosed that the amino acid release into the medium was slightly greater from the SFD than from the normal placenta, and also there was no significant difference in the release between serine and lysine. Considering no positive correlation observed between the $\mathrm{f} / \mathrm{m}$ ratio and the amino acid uptake in the placental slice, the actual results seem to point out technical problems in experiments rather than indicating the absence of idiosyncrasy in extracellular release mechanism of amino acids.

In the test to substantiate the probability that placental uptake of amino acids is done through the active transport, it is necessary to analyse the activity of membrane-bound Na-K ATPase, which is known to play a key role in amino acid transport. So far the fairly successful removal of the membrane has been reported for the erythrocytes, liver cells and free culture cells, but there are no reports available for the trophoblastic membrane perhaps because of difficulties in its isolation. In our present experiment, the trophoblastic membrane could be 
removed using Emmelot's method although it was not fairly pure. But the data are enough to suggest that the depressed activity of transport ATPase in the trophoblastic membrane had probably caused a decrease in uptake of amino acids in the SFD placenta.

Among reports on transport ATPase in the placenta available at present, Ishii et al. (1971) stated that the enzyme's activity is less in the placenta than in the erythrocytes, kidney and brain, and has no distinct difference between the early and term placentas. Performing more detailed analysis of the enzyme, Miller and Berndt (1973) stated that the placenta has at least two varieties of ATPase; one is $\mathrm{Ca}^{++}$-ATPase that concerns the transport of $\mathrm{Ca}^{++}$, and other is $\mathrm{Mg}^{++}$-dependent $\mathrm{Na}-\mathrm{K}$ ATPase which acts in the transport of amino acids and other organic compounds. Some of other authors' reports tend to support our review: Marton and Spearing (1972) in their electrophysiological research in the frog's skin, found a decrease in membrane potential with addition of pre-eclamptic serum; Lister (1966), in his electron-microscopic study on the placenta, suggested the phosphatase activity decreased in hypertensive pregnacy. Contrarily, Kuhnert et al. (1977), citing no change in ATPase activity in the pre-eclamptic placenta, suspected that the depressed amino acid transport in the pre-eclamptic placenta is likely to account for choline acetyl transferase activity.

For identifying the mechanism of amino acid transport in the placenta, including that of SFD, it seems necessary to pursue the role of transport ATPase, choline acetyl transferase, $\gamma$-glutamyl cycle, adenyl cyclase-cyclic AMP system, and their interrelationships.

\section{Acknowledgment}

We wish to thank the kind advices of Prof. K. Kojima, Department of Oncologic Pathology, Nara Medical University.

\section{References}

1) Christensen, H.N., Cespedes, C., Handlogten, M.E. \& Ronquist, G. (1973) Energization of amino acid transport, studies for the Ehrlich ascites tumor cell. Biochim. biophys. Acta (Amst.), 300, 487-522.

2) Dancis, J., Money, W.L., Springer, D. \& Levitz, M. (1968) Transport of amino acids by placenta. Amer. J. Obstet. Gynec., 101, 802-829.

3) Emmelot, P., Bos, C.J., Benedetti, E.L. \& Ruemke, P. (1964) Studies on plasma membranes. 1. Chemical composition and enzyme content of plasma membrane isolated from rat liver. Biochim. biophys. Acta (Amst.), 90, 126-145.

4) Enders, R.H., Judd, R.M., Donohue, T.M. \& Smith, C.H. (1976) Placental amino acid uptake. III. Transport systems for neutral amino acids. Amer. J. Physiol., 230, $706-710$.

5) Fiske, C.H. \& Subbarow, Y. (1925) Colorimetric determination of phosphorus. $J$. biol. Chem., 66, 375-400.

6) Gusseck, D.J., Yuen, P. \& Longo, L.D. (1975) Amino acid transport in placental slices. Mechanism of increased accumulation by prolonged incubation. Biochim. biophys. Acta (Amst.), 401, 278-284.

7) Ishii, S., Ikeura, T., Nakamura, I. \& Nagai. K. (1971) Some observations on the activity of transport ATPase in the human placenta. Wakayama med. Rep., 14, 133- 
138.

8) Kuhnert, B.R., Kuhnert, P.M., Murray, B.A. \& Sokol, R.J. (1977) Na/K- and MgATPase activity in the placenta and in maternal and cord erythrocytes of preeclamptic patients. Amer. J. Obstet. Gynec., 127, 56-60.

9) Lister, U.M. (1966) Ultrastructure of the placenta in hypertensive states. J. Obstet. Gynaec. Brit. Cwlth, 73, 439-451.

10) Litonjua, A.D., Canlas, M., Soliman, J. \& Paulino, D.Q. (1967) Uptake of $\alpha$-aminoisobutyric acid in placental slice at term. Amer. J. Obstet. Gynec., 99, 242-246.

11) Lowry, O.H., Rosenbrough, N.J., Farr, A.L. \& Randall, R.L. (1951) Protein measurement with the folin phenol reagent. $J$. biol. Chem., 193, 265-275.

12) Marton, I. \& Spearing, G. (1972) A factor in pre-eclamptic serum affecting sodium transport across a membrane. J. Obstet. Gynaec. Brit. Cwlth, 79, 40-43.

13) Matsuda, T., Nakano, Y., Nishikawa, Y. \& Yamaguchi, R. (1977) Feto-maternal amino acid patterns and cyclic AMP in the human placenta with abnormal pregnancies, particularly with SFD. Tohoku J. exp. Med., 121, 253-262.

14) Miller, R.K. \& Berndt, W.O. (1973) Evidence for $\mathrm{Mg}^{2+}$-dependent, $\mathrm{Na}^{+-} \mathrm{K}_{+}^{+}$-activated ATPase and $\mathrm{Ca}^{2+}$-ATPase in the human term placenta. Proc. Soc. exp. Biol. Med., 143, 118-122.

15) Miller, R.K. \& Berndt, W.O. (1974) Characterization of neutral amino acid accumulation by human term placental slices. Amer. J. Physiol., 227, 1236-1242.

16) Miller, R.K. \& Berndt, W.O. (1977) Mechanisms of transport across the placenta: An in vitro approach. Life Sci., 16, 7-30.

17) Nordlie, R.C. \& Arion, W.J. (1966) Phosphatase (III) Glucose-6-phosphatase. In: Methods in Enzymology, Vol. 9, edited by S.P. Colowick \& N.O. Kaplan,, Academic Press, New York-London, pp. 619-625.

18) Schneider, H. \& Dancis, J. (1974) Amino acid transport in human placental slices. Amer. J. Obstet. Gynec., 120, 1092-1098.

19) Smith, C.H., Adcock, E.W., Teasdale, F., Meschia, G. \& Battaglia, F.C. (1973) Placental amino acid uptake: Tissue preparation, kinetics, and pre-incubation effect. Amer. J. Physiol., 224, 558-564.

20) Sybulski, S. \& Tremblay, P.C. (1967) Uptake and incorporation into protein of radioactive glycine by human placentas in vitro. Amer. J. Obstet. Gynec., 97, 11111118. 